\title{
Stemming vision loss with stem cells
}

\author{
Valentina Marchetti, ${ }^{1}$ Tim U. Krohne, ${ }^{1}$ David F. Friedlander, ${ }^{2}$ and Martin Friedlander ${ }^{1}$ \\ 1Department of Cell Biology, The Scripps Research Institute, La Jolla, California, USA. ${ }^{2}$ School of Medicine, Vanderbilt University, Nashville, Tennessee, USA.
}

\begin{abstract}
Dramatic advances in the field of stem cell research have raised the possibility of using these cells to treat a variety of diseases. The eye is an excellent target organ for such cell-based therapeutics due to its ready accessibility, the prevalence of vasculo- and neurodegenerative diseases affecting vision, and the availability of animal models to demonstrate proof of concept. In fact, stem cell therapies have already been applied to the treatment of disease affecting the ocular surface, leading to preservation of vision. Diseases in the back of the eye, such as macular degeneration, diabetic retinopathy, and inherited retinal degenerations, present greater challenges, but rapidly emerging stem cell technologies hold the promise of autologous grafts to stabilize vision loss through cellular replacement or paracrine rescue effects.
\end{abstract}

Stem cell-based therapy represents a newly emerging therapeutic approach by which vascular and neuronal degenerative diseases may be treated. Since most of the diseases that lead to loss of vision do so as a result of abnormal vasculature and/or neuronal degeneration, the use of stem cells to stabilize or prevent visual loss may hold great promise. The eye is a highly vascular organ whose function is to gather light, focus it (through the cornea and lens) onto a thin, highly vascular neuronal tissue (the retina), transduce the light energy into electrical signals, transmit these signals via neurons to the visual cortex of the brain, and, finally, transform these electrical signals into an image or images that we perceive as "vision." Abnormalities in any part of the visual pathway, phototransduction machinery, neuronal pathways, or the visual cortex can lead to loss of vision. Most commonly, these abnormalities result from problems with the vasculature (e.g., ischemia or leakage) or the neurons themselves (e.g., genetic disease or degeneration secondary to hypoxia or toxicity), although ancillary structures of the eye that regulate intraocular fluid homeostasis (leading to glaucoma) or light transmission (leading to scarring of the front of the eye or cataract) can also be damaged sufficiently to lead to loss of vision. The potential clinical utility of stem cells could come from actual replacement of damaged cells with healthy ones generated from stem cells or through a paracrine effect of the stem cells that would help maintain a healthy tissue microenvironment or attract endogenous, circulating progenitor cells to help repair damaged cells in the eye. There has been enormous enthusiasm for exploring the potential utility of stem cells for a variety of diseases, including those of the eye, but much of this needs to be tempered by the reality of, first, needing to understand the underlying diseases and, second, finding the appropriate stem cell population and determining whether the potential benefit is outweighed by potential harm. In this article, we will discuss the ocular diseases potentially treatable with stem cell therapies, the various types of stem cells that may find therapeutic application, and the safety and production issues that need to be addressed before such therapies can be effectively used in the clinics.

The eye is divided into two anatomic regions (Figure 1), the anterior segment (containing the conjunctiva, cornea, trabecular meshwork [TM], and iris) and the posterior segment (consisting of the lens, vitreous, retina, and choroid/choriocapillaris). General pathological principles apply to the tissues of both segments, but

Conflict of interest: The authors have declared that no conflict of interest exists. Citation for this article: JClin Invest. 2010;120(9):3012-3021. doi:10.1172/JCI42951. inflammatory, infectious, and vascular diseases are more characteristic of diseases observed in the anterior segment while these three classes of disease plus degenerative/genetic problems are also observed in the posterior part. Certain parts of the eye (e.g., the retina) are highly vascular and, thus, more commonly experience diseases associated with abnormal blood vessels and associated cells (e.g., diabetic retinopathy [DR] and hypertensive retinopathies).

Stem cells are unspecialized cells capable of self renewal through cell division, and, under certain physiological or experimental conditions, they can be induced to become tissue- or organ-specific cells with special functions (1). Stem cells can be derived and/or obtained from tissues of early embryos or adults and, under appropriate conditions, will differentiate into more than one mature and functional cell type $(2,3)$. Stem cells have been identified in a variety of adult tissues as well as in cord and peripheral blood and adult BM. These cells represent a pool of progenitor cells that may serve to provide replacement cells critical to the maintenance of various tissue types. They could also be harnessed for the repair of damaged tissue following injury or stress. Stem cells that have the capacity to differentiate into any cell type are referred to as pluripotent; these are similar to cells obtained from the early dividing embryo (e.g., the epiblast tissue of the inner cell mass of a blastocyst or earlier morula stage embryos). Other types of stem cells may be more restrictive in their differentiation abilities, and a more restrictive destiny is characterized by a progenitor cell. Progenitor cells are a type of cell that has committed to a specific cell lineage, is capable of undergoing limited replication, and is not yet expressing the characteristics associated with the mature cell type.

Adult stem cells may have wide utility in the treatment of many eye diseases, as discussed below. In the appropriate microenvironment of many different tissues, these cells will target to sites of injury (vascular as well as neuronal), differentiate into various cell types, and contribute to the stabilization of degenerating tissue $(4,5)$. Newly emerging paradigms describing the existence of trophic "crosstalk" between local vascular networks and the tissues they supply and, thus, potential applications of these cells include not only cell-based therapeutic delivery of various trophic and static substances, but also use as stabilizing elements in an otherwise unstable environment of the type observed in ischemic retinopathies, retinal degenerative diseases, glaucoma, and corneal injuries. Here, we review newly emerging concepts and technologies in stem cell biology that have potential application to the treatment of vascular and neurodegenerative diseases of the eye. If successful, these studies will lead to the development and application of novel cell-based therapies for these currently untreatable diseases. 


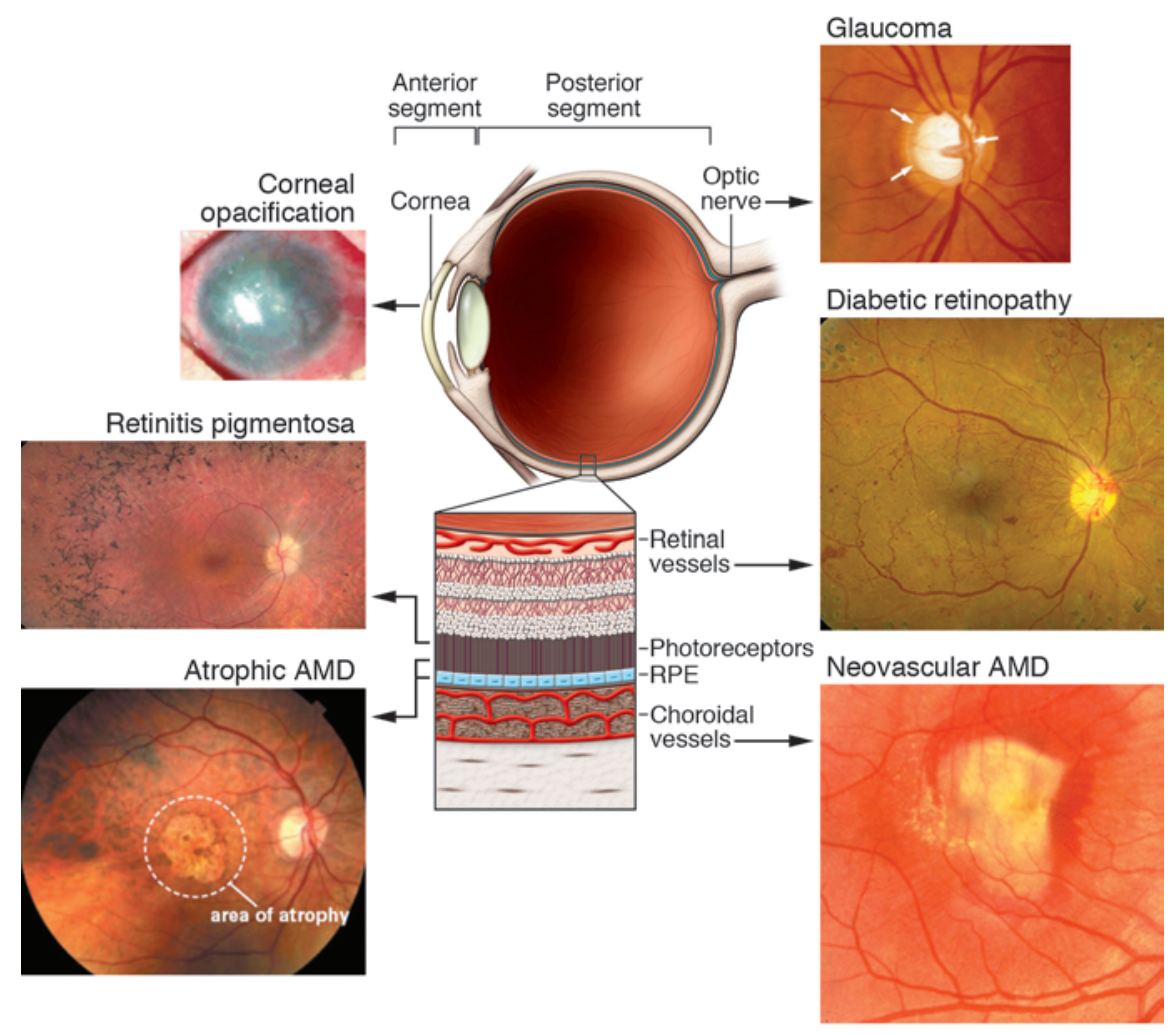

Figure 1

Schematic representation of the eye with images of diseases associated with different regions of the eye that may be amenable to treatment with stem cells. As discussed in the text, the eye can be divided into anterior and posterior segments with different diseases characteristic of these segments. Diseases that would be potentially treated with stem cell-based therapies include corneal opacification (image reproduced with permission from the New England Journal of Medicine; ref. 9) and glaucoma (enlarged optic nerve head cup/disc ratio). Glaucoma is typically considered a disease of the anterior segment, since treatments have historically been directed at decreasing fluid production or increasing fluid outflow by targeting the ciliary body or TM, respectively. The vision-threatening pathology, however, is in the posterior segment principally affecting the ganglion cells and optic nerve. In the posterior segment, degenerative neuronal diseases could include those affecting photoreceptors such as retinitis pigmentosa and the photoreceptors and retinal pigmented epithelium such as atrophic macular degeneration. Retinal vascular diseases of the posterior segment would include DR and neovascular macular degeneration.

\section{Stem cell therapies for the treatment of anterior segment diseases}

Stem cells for the treatment of limbal stem cell deficiency. The anterior segment of the eye consists primarily of the cornea and iris; it is separated from the posterior segment of the eye by the lens. Glaucoma and cataract represent the most prevalent anterior segment eye diseases within the US. Diseases affecting the cornea, such as hypersensitivity reaction-mediated Stevens-Johnson syndrome, also represent a significant source of visual loss (6). While surgical treatment of cataracts has proven to be highly efficacious (7), successful treatment of corneal disease remains elusive. The current mainstay of corneal disease therapy, corneal transplantation, is often complicated by chronic rejection of the transplanted tissue or structural abnormalities such as keratoconus - the cornea thins and assumes a conical shape, thereby distorting vision (8). Consequently, tissue replacement therapies concerned with anterior segment diseases have largely focused on utilizing corneal stem cells as either an adjunct to corneal transplantation or as a means of generating an entirely new cornea ex vivo $(9,10)$.
The healthy cornea is an avascular, transparent structure characterized by an external layer of stratified squamous epithelium, an intermediate area of alternating orthogonal layers of collagen, and an inner monolayer of endothelium. Schermer and colleagues (11) were the first to describe an endogenous corneal stem cell population in the limbus, the transitional area where the cornea and the sclera (the white of the eye) meet. These stem cells serve as a labile pool of renewable cells for the most superficial corneal epithelium, with vertical turnover ranging from 7-14 days in most mammals (12). When this process of cell renewal is altered by disease or physical/chemical trauma to the eye, such as that observed in infections, alkali burns, or severe trauma, the ability of the surface epithelium to transmit light and protect against environmental stresses becomes compromised. Equally as significant, depletion of the endogenous corneal stem cell population - as occurs in Stevens-Johnson syndrome and patients that have undergone multiple eye surgeries - can lead to conjunctival epithelial invasion into the cornea, resulting in functional blindness that cannot be treated with corneal transplantation (13).

Several groups have demonstrated the utility of corneal stem cell-mediated epithelialization of the cornea in treating either diseases where the integrity of the epidermal-dermal border is compromised (e.g., Stevens-Johnson syndrome and ocular pemphigoid) or the surface of the eye has been chemically injured $(10,14)$. In each of these conditions, limbally located corneal stem cells are depleted (a condition known as limbal stem cell deficiency [LSCD]), resulting in corneal scarring, opacification, and, ultimately, impaired vision. By transplanting donor limbal tissue enriched with corneal stem cells and allogenic donor cornea when necessary, Tsubota and colleagues were able to significantly improve visual acuity. Given the highly invasive nature of this procedure and concerns about rejection of the donor tissue, other groups have focused on the use of autologous corneal stem cell therapies in the treatment of LSCD (15). In this process, small biopsies of limbal epithelium, including limbal stem cells, are taken from the patient's healthy eye (if disease is unilateral) or the healthy eye of a living or cadaveric relative (for bilaterally affected patients) and expanded ex vivo on human amniotic membrane (HAM) or 3T3 fibroblasts treated with mitomycin C. This technique has many advantages over limbal tissue transplantation in that it requires less donor tissue and, in the case of autologous tissue grafting, eliminates the need for systemic immune suppression (15). Most recently, autologous corneal limbal stem cells cultivated and expanded on fibrin were used to successfully restore vision in three-fourths of contralateral eyes of patients with corneal damage resulting largely from burn injuries (16). 
Another approach that circumvents the need for invasive procedures used to harvest limbal tissue involves the use of autologous epithelial cells from the buccal mucosal (the mucus membrane of the inside of the cheek) as a source of corneal replacement tissue $(9,17)$. In this process, a sheet of buccal mucosa is surgically excised from the patient and subsequently expanded ex vivo on either mitomycin C-treated 3 T3 feeder cells or $\operatorname{HAM}(9,17)$. Given the logistical challenges posed by the need for readily available HAM and possible immunological issues associated with the use of nonhuman feeder cells, others have developed a technique that uses commercially available fibrin sealants to produce carrierfree sheets on which to expand the mucosal tissue $(18,19)$. While attractive from an immunological perspective, the long-term viability and regenerative capacity of buccal sheets grown on these substrates has yet to be determined and, as such, larger longitudinal clinical studies are needed to determine the long-term benefit of these procedures.

Recently, work from several laboratories has challenged the prevailing notion that the limbus is the sole niche of corneal stem cells. Majo et al. demonstrated that stem cell populations in the pig eye are not limited to the limbus, but rather can be found throughout the ocular surface, including the cornea (20). Under appropriate culture conditions, these cell populations were shown to have the capacity to generate individual colonies of corneal and conjunctival cells in vitro (20).

While tissue replacement therapies utilizing corneal stem cells hold high therapeutic potential for diseases affecting the surface of the eye, many of the surgical techniques by which cultured tissue is delivered to the recipient eye have marginal success rates of reepithelialization. Even when epithelialization does occur, improvements in visual acuity are often hampered by postsurgical complications such as graft rejection and ocular hypertension, which have been shown to occur in $46 \%$ and $37 \%$ of patients, respectively (9). While autologous sources of limbal tissue may reduce the likelihood of transplant rejection, the use of undefined animal products (such as fetal bovine serum) and feeder cells in the ex vivo expansion process represent potential immunogenic obstacles to successful tissue transplantation. While such concerns have been partially addressed by using autologous serum and feeder cell layers of human origin (21), the relative efficiency of these techniques remains to be seen (21). Another consideration is the possible depletion of limbal corneal stem cells in the healthy donor eye, following successive rounds of cell harvesting (22). As discussed above, several investigators $(9,17)$ have demonstrated that buccalderived epithelial sheets represent an alternative source of corneal epithelium if the individual's limbal stem cell population has been depleted. Future research in the field of corneal replacement therapy must address these issues. Consequently, future work likely to lead to significant improvements within the field will focus on autologous progenitor cell sources, such as the buccal mucosa, cultivated in chemically defined culture conditions.

Glaucoma. Individuals suffering from glaucoma, whereby increased intraocular pressure leads to ganglion cell damage and ultimately vision loss, might also benefit from advances in stem cell biology. Several groups have begun to explore potential therapeutic applications of stem cells derived from the TM, which comprises the tissue through which intraocular fluid leaves the eye (23). It has long been known that a decrease in TM cellularity accompanies the onset of primary open angle glaucoma (POAG) $(24,25)$. POAG is typically characterized by an increase in intra- ocular pressure that can lead to visual field loss and blindness as a result of ganglion cell death and optic nerve atrophy. While the precise etiology of POAG is still unknown, one possible mechanism is obstruction to outflow of intraocular fluid resulting from malfunction of the TM, including the Schlemm canal (26). The discovery of a putative stem cell-like population of TM cell progenitors (termed "insert cells" due to their proximity to the area where the TM inserts into the cornea) by Acott and others raised the prospect of expanding and ultimately implanting these cells into the TM of glaucomatous eyes (27-29). While several groups have successfully isolated putative TM progenitor cell populations from free-floating neurospheres derived from human TM cells (30), phenotypic differences between these cells and mature TM cells do exist (31). Little progress has been made with regard to applying these cells clinically.

Another approach to using stem cells to replace defective cells involved in glaucoma would be to derive ganglion cells from various progenitors, since the primary cell type affected in glaucoma is the ganglion cell. While this has a certain appeal at the biological level, it may be difficult to "rewire" the nerve fiber layer containing the ganglion cells once they have been irreparably damaged by the sustained increased intraocular pressures associated with glaucoma. Nevertheless, several groups have demonstrated that it may be possible to derive retinal ganglion cells from human-induced pluripotent $(32,33)$ and embryonic (34) stem cells.

\section{Stem cells and therapy of retinal vascular diseases}

The posterior segment of the eye consists of the ocular structures behind the lens, primarily the vitreous (the transparent, colorless gel found between the lens and the retina) and the retina (Figure 1). Some of the most common diseases that lead to vision loss in industrialized nations do so as a result of retinal vascular abnormalities in the posterior segment of the eye. These diseases include $\mathrm{DR}$ and retinopathy of prematurity (ROP). Other, less common diseases of the retina such as macular telangiectasia (MacTel) and retinal angiomatous proliferation (RAP) involve focal vascular abnormalities that may also be associated with neuronal (e.g., cone photoreceptor) loss. "Crosstalk" between local vascular networks and the tissues they supply may help maintain physiological homeostasis and function in a variety of tissues including the retina (35-37). In fact, endothelial cells are known to provide trophic substances that greatly stimulate self renewal and directed differentiation of neural stem cells in the central nervous system (38) as well as hepatocytes during liver regeneration in response to injury (39). Given such interdependency of vascular endothelial cells and surrounding tissues, it may be possible to utilize progenitors of either vascular or neuronal cells to provide a paracrine rescue function in the face of severe stress such as hypoxia or genetically encoded cell-specific degenerations. Such progenitor cells may be more resistant to hypoxia and/or oxidative damage and exert a trophic rescue effect on local populations of vascular and neuronal cells. Such a therapeutic approach would obviate the need to employ destructive treatment modalities and would facilitate repair of damaged and maintenance of normal retinal tissue.

Stem cell-based therapy is associated with numerous events that involve both the injected stem cells and cellular/extracellular matrix microenvironment into which they are injected (40). The retinal $(41,42)$ and subretinal $(43)$ tissue microenvironments can modulate the functionality of injected autologous or allogeneic cells by inducing differentiation to several different cell types. 
A

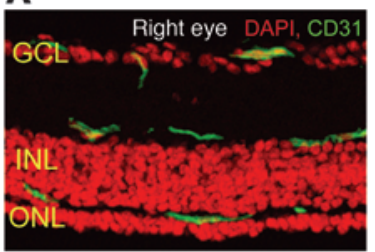

B

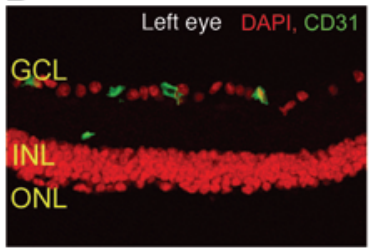

Figure 2

BM-derived Lin- HSC injection rescues the degeneration of vascular and neural cells in a mouse model of retinal degeneration ( $\mathrm{rd} 1 / \mathrm{rd} 1)$. Retinal vasculature (green, stained for CD31) is well preserved in the Lin--treated eye (A) and is degenerated except for small numbers of the superficial plexus in control-treated eyes (B). Neurotrophic rescue is also observed whenever the vasculature is rescued; in the controltreated eye (B), the outer nuclear layer (ONL) is completely degenerated and the inner nuclear layer (INL) atrophic. Retinal function is also preserved after Lin- HSC. GCL, ganglion cell layer. From the $\mathrm{JCl}(66)$.

Recent studies have employed small molecules, coinjected with the stem cells or administered to the cells prior to injection, to drive cells along specific differentiation pathways (44). These freshly differentiated cells could then serve as "replacement cells" for damaged local cells and/or exert paracrine functions by producing cytokines, growth factors, and other trophic molecules (37). Two other advantages to generating these replacement cells locally from injected progenitor cells are that less ex vivo manipulation of the donor cell population is required and that there is greater likelihood that the appropriately differentiated cells would be generated as a result of being in the appropriate microenvironment.

Adult BM-derived progenitors differentiate into endothelial progenitor cells and target activated astrocytes and provide vasculo- and neurotrophic rescue. Adult BM is a rich source of HSCs and hematopoietic progenitor cells (HPCs) $(35,36,38)$. These undifferentiated cells are able to differentiate into various cell types including myeloid and endothelial cells. The lineage-negative $\left(\mathrm{Lin}^{-}\right)$population of BM cells, which was first identified and purified from mouse BM, cannot differentiate into blood cells, a property of $\mathrm{Lin}^{+}$HSCs. Lin- HSCs are described as a heterogeneous population of progenitor cells that include cells able to differentiate into vascular endothelial cells and form blood vessels (endothelial progenitor cells [EPCs]) (45). The Lin ${ }^{-}$population from BM, as well as a population of progenitor cells derived from human cord blood, have, under specialized conditions, been observed to differentiate into cells expressing neuronal markers after transplantation into the subretinal space $(46)$ or brain $(47,48)$ in mice, although others claim that this is actually a result of transdifferentiation rather than true stem cell differentiation $(49,50)$. Others have explored the potential utility of human cord blood-derived mesenchymal stem cells as a source of retinal neuronal cells, but did not observe differentiation of these cells into neuronal-like elements (51). Human BM-derived CD45-/glycophorin ${ }^{-}$cells will differentiate into photoreceptors in vitro after being cocultured with retinal pigmented epithelial cells (52).

EPCs are mobilized from the BM in response to a variety of signaling molecules $(53,54)$ and target sites of angiogenesis in ischemic peripheral vasculature (45), myocardium (55), and injured eyes (56). Interestingly, BM contains several populations of progenitor cells that can differentiate into a variety of other cell types $(57,58)$. It has been shown that HSCs contain a pool of progenitor cells that can directly differentiate into EPCs and other progenitor cells that, through a paracrine effect, can promote stabilization of endothelial cells, although there is continuing controversy as to the precise identity of these cells and whether they facilitate vascular normalization or enhance pathological neovascularization under conditions of hypoxic stress (59-61). While there is a large, and still growing, literature on the potential therapeutic benefits of EPC treatments for ischemic diseases, the definition, identification, purification, and characterization of the EPC remains elusive as does the role for these cells in the maintenance of vascular homeostasis and disease (52-55). Without a standard set of in vitro and in vivo assays that associate specific cell phenotypic profiles with specific activities, the EPC remains poorly defined. After the first description of an adult endothelial precursor cell (45), multiple studies have characterized, phenotyped, and functionally analyzed endothelial precursors. Today the term EPC describes a heterogeneous group of cells including circulating and culture-differentiated cells that have the capacity to differentiate into endothelial cells. Protocols for obtaining these cells are as heterogenous as the cells themselves. There is evidence that EPCs can participate in early phases of vascular damage (59-61) and that this particular population of EPCs has monocyte-like features (62). Monocyte-derived EPCs adhere to lesion sites and differentiate into endothelial cells that facilitate vascular stabilization and repair. In the later phase of vascular repair, these cells may recruit, in a paracrine fashion, other EPC types and further facilitate vascular repair (63).

In 2004, Otani et al. demonstrated that BM-derived EPCs injected directly into the vitreous of neonatal mice stably incorporated into forming blood vessels, forming vascular mosaics of the injected EPCs and endogenous retinal vascular endothelial cells (64). The injected EPCs were observed to target to retinal astrocytes, which form a template over which the developing endogenous retinal vasculature develops and are dependent on R-cadherin-mediated adhesion for proper targeting (65) If the $\mathrm{Lin}^{-}$progenitor cells are injected in the vitreous of mice with retinal degeneration caused by a genetic mutation, they completely prevent the retinal vascular degeneration observed in untreated animals and also rescue the neuronal retinal degeneration (Figure 2) (66). If these cells are engineered to express an angiostatic fragment of tryptophan tRNA synthetase (T2-trpRS) prior to intravitreal injection, they will potently inhibit neovascularization in neonatal mouse retinal angiogenesis $(64,67)$. The use of stem cells as cell-based delivery systems has the advantage over more traditional systemic drug administration in that such cell-based delivery permits the selective, potent delivery of drugs to the back of the eye in physiological doses.

Adult BM-derived myeloid progenitor cells. Recent data support the concept that myeloid cells are involved in the regulation of angiogenesis and neovascularization, independent of the effects observed for EPCs (68). Myeloid progenitor cells have been reported to rescue and maintain the function of ischemia-damaged endothelial cells of the hind limb (69) and to prevent vascular abnormalities in a mouse model of ischemic injury, oxygeninduced retinopathy (OIR) (68). In this model, a subpopulation of Lin $^{-}$cells that express high levels of the hyaluronic acid receptor, CD44, enhances vascular repair following oxygen-induced vascular obliteration and stabilizes hypoxia-driven neovascularization. The high level of expression of myeloid-specific markers, cellular morphology, and the localization of the cells external to the lumen of the blood vessels define these cells as microglia. There is a growing interest in the role of microglia in maintaining vascular function and homeostasis during normal and stress-associated 
conditions in the retina where they provide a vasculoprotective function (70) and in the brain where BM-derived, vasculatureassociated microglia cells have been observed in noninjured adult tissue (71). Activated microglia and microglial perivasculitis have been observed in association with human DR and may play a role in the development of vascular and neuronal changes observed in this disease (72), suggesting that manipulating vasculature-associated microglia or their progenitors may be useful for the treatment of ischemic retinopathies of the retina and brain.

Cord blood as a source of progenitor cells. Human umbilical cord blood (UCB) is a well-described source of HSCs used for transplantation in the treatment of hematological and genetic diseases $(73,74)$. Since the first human transplantation in 1988, many studies have contributed to the extensive characterization of these progenitor cells. Compared with adult BM, cord blood contains a much higher concentration of HSC and these cells have a greater capacity to form hematopoietic colonies and differentiate into multiple blood cell lineages. Clinical observations suggest that patients transplanted with UCB-derived HSCs have a relatively low incidence of graft-versus-host disease (GVHD), likely due to the lower immunogenicity of these cells as compared with those derived from adult BM (75).

EPCs derived from human UCB were observed to be a heterogeneous population of cells expressing CD34 and CD11b. When UCB-derived EPCs were grown in culture, they upregulated expression of endothelial markers such as Tie2 and Ang1 $(76,77)$. This differentiation potential suggests that stem cells circulating in cord blood may not only give rise to hematopoietic cells but may also have therapeutic application for the repair of vascular endothelium following injury and surgery. Thus, UCB-derived cells that can regulate inflammation cascades and hypoxia-driven neovascularization may be useful for the treatment of diseases such as ROP and DR, in which an early phase of vaso-obliteration is followed by inflammation-associated neovascularization (78). The fact that cord blood is much easier to obtain than BM from a newborn child, and that cord blood is a less immunocompetent source of cells than peripheral blood and adult BM, makes the use of this stem cell source for the treatment of retinopathies such as ROP very appealing.

In recent years, the importance of circulating monocytes/ macrophages in neovascularization has been demonstrated for ischemic diseases of the myocardium $(79,80)$. Monocytes, which are derived from monoblasts (the HSC precursors in BM) circulate in the bloodstream before extravasating into tissues of the body and promoting angiogenesis related to inflammatory reactions (81). Monoblasts and their monocytic progeny are attracted to hypoxic areas and begin to differentiate to tissue macrophages, dendritic cells, and microglia.

Resident tissue macrophages have been known to exist as polarized populations, M1 and M2 subsets $(82,83)$. While M1 macrophages are proinflammatory and phagocytose pathogens, M2 macrophages modulate the inflammatory response and help with angiogenesis and tissue repair. Recent reports confirm that most monocytes from UCB become M2 polarized cells (84, 85 ), observations that could be explained by the immaturity of the immune and inflammation stimulatory functions of UCB. For these reasons, cord blood-derived myeloid progenitor cells/ monocytes and macrophage progeny may provide a promising therapeutic option for the treatment of ischemic ocular diseases such as DR or ROP. These progenitor cells may facilitate physiological revascularization through their ability to promote angiogenesis without associated inflammation.

\section{Stem cell therapy of neurodegenerative retinal diseases}

Age-related macular degeneration as a target for stem cell-based therapy. In contrast to vascular retinopathies that affect the retinal vasculature and only secondarily result in neuronal damage, most degenerative and hereditary retinopathies primarily target the functional complex of photoreceptors and supporting retinal pigment epithelium (RPE). Thus, the current development of stem cell-based therapies for these diseases mostly focuses on rescuing, supplementing, or replacing these two cell types in the affected retina. Age-related macular degeneration (AMD) is by far the most common of these retinopathies and, indeed, represents the most common blinding disease in general, accounting for about half the cases of legal blindness in all developed countries $(86,87)$. Moreover, this already high prevalence is projected to increase substantially in the coming decades as a consequence of demographic changes (88).

Despite significant progress in recent years, the multifactorial pathogenesis of AMD has not yet been fully elucidated. However, several lines of clinical and experimental evidence indicate that progressive dysfunction of the aging RPE initiates a pathogenetic cascade that eventually results in visual decline $(89,90)$. The singlelayered RPE is located at the interface between photoreceptors and the underlying vascular layer of the eye, the choriocapillaris. It performs critical tasks vital for photoreceptor function and survival such as retinoid recycling and fluid transport (91). Thus, RPE cells and photoreceptors form a functional unit, and RPE cell degeneration inevitably results in secondary loss of photoreceptor cells.

The two blinding late-stage manifestations of AMD include atrophic AMD, which is characterized by patchy atrophy of RPE in the central retina and degeneration of associated photoreceptors, and neovascular AMD, in which VEGF-mediated choroidal neovascularization $(\mathrm{CNV})$ into the outer retina damages photoreceptors primarily by fluid leakage and chronic retinal edema formation. Despite extensive research efforts, treatments currently are not available for atrophic AMD and are highly limited for neovascular AMD. Recently introduced VEGF-blocking drugs provide for the first time a therapeutic option to stop disease progression in a subset of patients with neovascular AMD, but the effect is only temporary and requires repeated intraocular injections to maintain it $(92,93)$. Surgical removal of CNV membranes in neovascular AMD is feasible and has been shown to effectively stop neovascular growth; however, the procedure inevitably results in an RPE defect at the site of the CNV extraction that precludes visual recovery.

Transplantation of RPE cells by subretinal injection has been evaluated in humans, but led to graft rejection when allogeneic cells were used $(94,95)$. While the subretinal space is relatively immune privileged, this privilege is not absolute and systemic immunosuppression would be necessary to maintain an allogenic graft; recent advances in the development of implantable sustained release devices (96) may provide more effective immunosuppression without systemic side effects. To circumvent the need for allogeneic grafts, different surgical approaches for autologous RPE replacement have been tested in clinical trials. These include translocation of the central retina to an area of viable RPE by 360 degree retinal dissection and complete retinal detachment (97) as well as excision of an RPE patch from the retinal periphery, with subsequent transplantation under the central retina of the same eye (98). While the clinical applicability of these procedures may be limited due to the significant complication rate resulting from their highly invasive nature, these studies demonstrated the feasibility and potential effectiveness of autologous RPE replacement therapy in atrophic and neovascular AMD. 


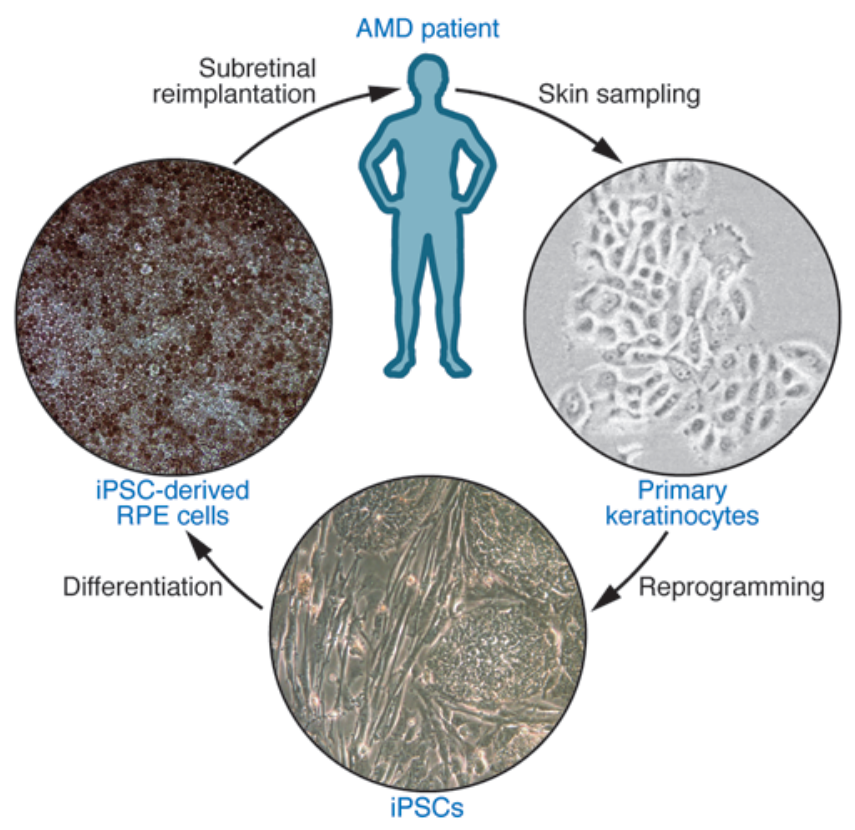

Utilizing embryonic stem cells for RPE replacement therapy. Several groups have evaluated embryonic stem cells (ESCs) for differentiation of RPE cells and tested their effects on visual function after transplantation into animal models of RPE-mediated retinal degeneration, such as the Royal College of Surgeons (RCS) rat (99-103). Untreated long-term cultures of human ESCs were observed to reproducibly generate cells that exhibited characteristic RPE morphology and expression of markers specific for RPE cells, such as RPE-specific protein 65 kDA (RPE65), cellular retinaldehyde-binding protein (CRALBP), and bestrophin (99-103). Subretinal injection of human ESC-derived RPE cells into RCS rats resulted in cell survival and integration into the RPE as well as temporary rescue of retinal degeneration and visual decline (100, 101). However, spontaneous differentiation of ESCs is a lengthy and inefficient process. To advance this approach toward clinical applicability, different groups have successfully developed culture techniques that reduce the time and increase the yield of RPE differentiation, either by coculture with feeder cells that possess neurodifferentiating properties (104) or by treatment with substances such as activin A, Dkk1, and Lefty-A that mimic certain aspects of embryonic RPE development (105-107). Cells generated using these protocols have also demonstrated rescue effects in animal models of RPE degeneration (105).

Generation of autologous RPE grafts from induced pluripotent stem cells. Studies using ESCs provide important insights regarding the feasibility of stem cell-derived RPE transplantation. For clinical application, however, induced pluripotent stem cells (iPSCs), derived from adult somatic cells by genetic reprogramming using either the four transcription factors Octamer 3/4 (Oct4), SRY box-containing gene 2 (Sox2), Krüppel-like factor 4 (Klf4), and cellular myelocytomatosis oncogene (c-Myc) (108), or Oct4, Sox2, Nanog, and Lin28 (109), may be preferable because they could be generated from the patient's own somatic cells (Figure 3 ). While the subretinal space in the healthy eye is relatively immune privileged due to the blood-retinal barrier function of the RPE layer, disruption of this barrier with RPE cell loss in a disease such as AMD may abolish the immune privilege and result in rejection of

\section{Figure 3}

Example of a potential treatment strategy for degenerative/dystrophic retinopathies using autologous iPSCs. Skin cells such as keratinocytes or fibroblasts are sampled from the patient by biopsy and expanded ex vivo. Reprogramming into iPSCs is achieved by conventional protocols using retroviral transfection or, preferentially, by alternative protocols that employ nonintegrating vectors or vector-free treatment with small molecules. Directed differentiation regimens are used to derive the required type of fully differentiated retinal cells, such as RPE or photoreceptors, from the iPSC cultures. Following further enrichment and expansion steps, retinal cells will be reimplanted directly into the subretinal space during intraocular surgery. By supplementing or replacing diseased cells, the transplanted cells may prevent further disease progression and visual decline. RPE cells generated from human 4-factor iPSCs have characteristic polygonal shapes, express ZO-1 junction proteins, and vectorially transport fluid, as evidenced by "dome" formation.

allogenic grafts such as ESC-derived RPE. Hence, transplantation of autologous, iPSC-based grafts could avoid not only the need for life-long immunosuppressive prevention of graft rejection but also the ethical and practical problems associated with large-scale clinical use of ESCs. Similar to ESCs, human iPSCs differentiate spontaneously into RPE cells $(102,110,111)$, and differentiation efficiency can be enhanced by manipulation of developmental signaling pathways with compounds such as Dkk1 and Lefty-A $(107,112)$. Analogous to RPE cells in vivo, iPSC-derived cells in vitro form monolayers with intercellular tight junctions, exhibit RPE-characteristic morphology and pigmentation, express RPEspecific markers, and are capable of photoreceptor outer segment phagocytosis (Figure 3). Moreover, they maintain most of these morphological and functional properties following subretinal transplantation into RCS rats and provide temporary retinal rescue and preservation of visual function (111).

Safety concerns regarding the clinical application of iPSCs arise from the potential risk of malignant transformation that results from the oncogenic properties of the transcription factors required by current reprogramming protocols, in particular c-Myc, as well as the random genomic integration of these factors following retroviral transduction. Extensive research efforts are underway to establishing new reprogramming strategies that use nonintegrating gene delivery approaches (113) or that replace the introduction of exogenous reprogramming factors by treatment with proteins (114) or small molecules (115). Meanwhile, iPSCs have also been successfully derived from human somatic cells that were reprogrammed using only three or two factors (Oct4/Sox $2 / \mathrm{Klf} 4$ or Oct4/Klf4, respectively) (115, 116). Eventually, iPSCs entirely free of genomic and epigenetic alterations would be desirable to minimize the tumor risk after transplantation therapies. Finally, transplantation of ESC- or iPSC-derived RPE cells into the microenvironment of a diseased retina, as that seen in macular degeneration, may compromise the ability of these newly grafted cells to achieve a therapeutic effect due to rapid compromise of the transplanted cells secondary to global dysfunction in the diseased tissue. 
Therapeutic photoreceptor cell regeneration using stem cells. AMD may be an ideal target for a stem cell-based therapeutic approach for a number of reasons. First, RPE cells can consistently be differentiated from stem cells. Second, AMD is not caused by monogenetic defects and only manifests late in life, meaning that autologous iPSC-derived RPE grafts would not be expected to be rapidly affected by the disease. Third, RPE cell grafts can be readily delivered directly into the correct location under the retina. Finally, unlike neuronal grafts, the transplanted cells do not require synaptic integration into the neuronal retinal network to become functional. In addition to AMD, however, hereditary retinopathies such as retinitis pigmentosa may also benefit from stem cell treatments. These diseases mostly result from monogenetic defects that directly cause photoreceptor degeneration and would therefore require therapeutic cell replacement with functional photoreceptor cells. Compared with RPE cells, photoreceptor differentiation in vitro has proven to be considerably more complex, and while photoreceptor progenitors have been successfully derived from human ESCs as well as iPSCs $(102,106,112)$, evidence for the generation of fully functionally photoreceptors is still lacking. The retina has been shown to contain a population of endogenous adult stem cells in the ciliary margin zone that can also be utilized for photoreceptor differentiation in vitro $(117,118)$, but the clinical utility of these stem cells, in particular for autologous transplantation, seems limited by their poor accessibility and small number. Indeed, the origin and nature of these ciliary margin zone-derived cells has been called into question (119). In a promising alterative therapeutic approach, transplantation of human neural progenitor cells has been used to rescue, rather than replace, degenerating photoreceptors in RCS rats (120). This effect was mediated by the release of neurotrophic factors and could be further enhanced when the cells were genetically modified to overexpress these factors, a finding that raises the intriguing possibility that stem cells may be useful as vehicles for drug delivery to the retina.

\section{Concluding remarks}

Stem cell-based therapy represents a newly emerging therapeutic approach by which vascular and neuronal degenerative diseases may be treated. Intravitreal injection of endothelial and myeloid progenitor cells prevents vascular regression and protects neurons in mouse models of retinal degeneration (66). Injection of myeloid progenitors facilitates vascular repair in models of ischemic retinopathy by accelerating normal revascularization of the superficial and deep retinal vascular plexuses and decreasing intravitreal vascular pathogenesis (68). These cell-based therapies have the potential to correct the underlying vascular abnormalities associated with ischemic retinopathies rather than simply treat the complications resulting from abnormal neovascularization. While we have gained some insight regarding the vasculo- and neurotrophic properties of these cells, the precise mechanism by which they facilitate vascular rescue in models of ischemic retinopathy remains unclear. These studies also raise many interesting questions surrounding the role of endogenous glial and inflammatory cells in retinal response to injuries such as hypoxia or oxidative stress. Clearly, microglia, astrocytes, and Mueller glia (a specialized giant glial cell unique to the retina that spans the entire retina and performs a myriad of functions, including the regulation of fluid transport and neurotrophic activity) may be critical to maintenance of the normal vascular and neuronal microenvironment in the retina. In addition, they represent attractive therapeutic targets, both as cell-based delivery devices (121) and as targets for modulation themselves. The major challenges in translating the application of endothelial and myeloid progenitor cells into the clinic remain better characterization of the various populations of progenitor cells and engineering a process for the purification, characterization, and infusion of these cells into patients. There are several centers of "stem cell tourism" that currently offer BM-derived progenitor cells for the treatment of several diseases, including those of the eye, but none offer procedures that are approved by recognized regulatory agencies nor are there any random, prospective, placebo-controlled study data to support these centers' claims of therapeutic efficacy and safety (122). Without documented efficacy and safety data, it is difficult to evaluate what, if any, benefit would be obtained for vulnerable patients subjecting themselves to the treatments offered.

It is now possible to generate pluripotent stem cells from adult somatic tissues and, thus, provide autologous grafts of various tissue types to enhance or replace damaged cells in nearly every organ in the body including the eye. While the eye is a relatively immune-privileged site, this privilege is not absolute and is compromised under many of the disease conditions that we would like to treat with stem cell therapies. Thus, the use of autologous grafts would be preferable to using stem cell-derived "replacement parts" from ESCs. Scale-up and treatment cost considerations may favor developing cell banks derived from ESCs, but if these grafts are ultimately rejected or induce inflammation, there will have been no gain. ES- or iPS-derived cells for replacing diseased neurons (e.g., photoreceptors, ganglion cells) may be technically feasible in the near future, but there will always be the problem of "rewiring" these neurons if placed into adult tissues with already established retinotectal projections that are notoriously difficult (if not impossible) to reestablish once there is retrograde degeneration of the type observed in chronic retinal generative disease (123).

The greatest opportunity to treat these neurodegenerative diseases of the eye (such as retinitis pigmentosa) will come from early intervention with cells providing trophic rescue of existing, albeit "sick," neurons. In this regard, one of the most potentially efficacious approaches may be to replace and/or rescue diseased RPE cells in patients with AMD. Generating RPE from human ESCs to serve as healthy grafts to replace diseased RPE in patients with AMD has merit, but there are limitations in using ESCs, since there is considerable variability in the quality of the RPE obtained from different human ESC lines and using fresh fetal material has obvious limitations. More importantly, the RPE obtained from human ESCs would not be autologous and would require growth on mouse feeder cell layers and/or Matrigel, conditions shown to be associated with retained murine antigenicity. As discussed above, one way to circumvent long-term systemic immunosuppression following RPE transplantation is to use autologous somatic cells induced to form pluripotent stem cells (i.e., iPSCs) from which the RPE could be derived. Differentiation of RPE from iPSCs is not without potential downside, as discussed above. To overcome these hurdles, increase the efficiency of generating iPSC-derived $\mathrm{RPE}$, and to avoid using lentiviral vectors, small molecules from unique chemical libraries and/or an episomal expression vector that simultaneously expresses the four human factors necessary to induce iPSCs from somatic cells may represent a path forward. There are several groups with early clinical development programs for the use of stem cells to treat retinal disease and as work in this area progresses, we will learn whether or not the promise of this exciting technology will be fulfilled. 


\section{Acknowledgments}

We would like to thank all the members of the Friedlander laboratory for their helpful comments on this manuscript and their contributions to the work cited from our laboratory. We also apologize to other investigators whose work may not have been referenced due to space constraints. We are very grateful for generous support of the work in our laboratory from the NIH (NEI R01 EY11254 and NEI R24 EY017540 to M. Friedlander), the California Institute for Regenerative Medicine (TR1-01219 to M. Friedlander), the Lowy Institute for
Biomedical Research (MacTel), and the V. Kann Rassmussen Foundation. T.U. Krohne is supported by the German Research Foundation (DFG; grant KR 2863/6-1). D.F. Friedlander was supported by a summer research fellowship from Vanderbilt University School of Medicine.

Address correspondence to: Martin Friedlander, The Scripps Research Institute, 10550 N. Torrey Pines Road, La Jolla, California 92014, USA. Phone: 858.784.9138; Fax: 858.784.9135; E-mail: friedlan@scripps.edu.
1. Weissman IL. Translating stem and progenitor cell biology to the clinic: barriers and opportunities. Science. 2000;287(5457):1442-1446.

2. Zhou Q, Melton DA. Extreme makeover: converting one cell into another. Cell Stem Cell. 2008; 3(4):382-388.

3. Slack JM. Origin of stem cells in organogenesis. Science. 2008;322(5907):1498-1501.

4. Li L, Clevers H. Coexistence of quiescent and active adult stem cells in mammals. Science. 2010; 327(5965):542-545.

5. Moore KA, Lemischka IR. Stem cells and their niches. Science. 2006;311(5769):1880-1885.

6. National Eye Institute. Prevalence of Blindness Data Tables. NIH Web site. http://www.nei.nih. gov/eyedata/pbd_tables.asp. Updated October 2008. Accessed July 19, 2010.

7. McKee M, Whatling JM, Wilson JL, Vallance-Owen A. Comparing outcomes of cataract surgery: challenges and opportunities. J Public Health (Oxf). 2005;27(4):348-352.

8. Claesson M, Armitage WJ, Fagerholm P, Stenevi $\mathrm{U}$. Visual outcome in corneal grafts: a preliminary analysis of the Swedish Corneal Transplant Register. Br J Ophthalmol. 2002;86(2):174-180.

9. Nishida K, et al. Corneal reconstruction with tissue-engineered cell sheets composed of autologous oral mucosal epithelium. $N$ Engl J Med. 2004;351(12):1187-1196.

10. Tsubota K, et al. Treatment of severe ocular-surface disorders with corneal epithelial stem-cell transplantation. $N$ Engl J Med. 1999; 340(22):1697-1703

11. Schermer A, Galvin S, Sun TT. Differentiationrelated expression of a major $64 \mathrm{~K}$ corneal keratin in vivo and in culture suggests limbal location of corneal epithelial stem cells. J Cell Biol. 1986;103(1):49-62.

12. Haddad A. Renewal of the rabbit corneal epithelium as investigated by autoradiography after intravitreal injection of $3 \mathrm{H}$-thymidine. Cornea. 2000;19(3):378-383.

13. Puangsricharern V, Tseng SC. Cytologic evidence of corneal diseases with limbal stem cell deficiency. Ophthalmology. 1995;102(10):1476-1485.

14. Kenyon KR, Tseng SC. Limbal autograft transplantation for ocular surface disorders. Ophthalmology. 1989;96(5):709-722.

15. Kolli S, Ahmad S, Lako M, Figueiredo F. Successful clinical implementation of corneal epithelial stem cell therapy for treatment of unilateral limbal stem cell deficiency. Stem Cells. 2010; 28(3):597-610.

16. Rama P, Matuska S, Paganoni G, Spinelli A, De Luca M, Pellegrini G. Limbal stem-cell therapy and long-term corneal regeneration. $N$ Engl J Med. 2010;363(2):147-155

17. Nakamura T, et al. The successful culture and autologous transplantation of rabbit oral mucosal epithelial cells on amniotic membrane. Invest Ophthalmol Vis Sci. 2003;44(1):106-116.

18. Higa K, et al. Proliferation and differentiation of transplantable rabbit epithelial sheets engineered with or without an amniotic membrane carrier.
Invest Ophthalmol Vis Sci. 2007;48(2):597-604.

19. Rama P, et al. Autologous fibrin-cultured limbal stem cells permanently restore the corneal surface of patients with total limbal stem cell deficiency. Transplantation. 2001;72(9):1478-1485

20. Majo F, Rochat A, Nicolas M, Jaoude GA, Barrandon Y. Oligopotent stem cells are distributed throughout the mammalian ocular surface. Nature. 2008;456(7219):250-254.

21. Nakamura T, et al. The use of autologous serum in the development of corneal and oral epithelial equivalents in patients with Stevens-Johnson syndrome. Invest Ophthalmol Vis Sci. 2006;47(3):909-916.

22. Chen JJ, Tseng SC. Corneal epithelial wound healing in partial limbal deficiency. Invest Ophthalmol Vis Sci. 1990;31(7):1301-1314.

23. Kelley MJ, Rose AY, Keller KE, Hessle H, Samples JR, Acott TS. Stem cells in the trabecular meshwork: present and future promises. Exp Eye Res. 2009;88(4):747-751.

24. Alvarado J, Murphy C, Juster R. Trabecular meshwork cellularity in primary open-angle glaucoma and nonglaucomatous normals. Ophthalmology. 1984;91(6):564-579.

25. Liton PB, Challa P, Stinnett S, Luna C, Epstein DL, Gonzalez P. Cellular senescence in the glaucomatous outflow pathway. Exp Gerontol. 2005; 40(8-9):745-748.

26. Moses RA. The effect of intraocular pressure on resistance to outflow. Surv Ophthalmol. 1977; 22(2):88-100.

27. Acott TS, Samples JR, Bradley JM, Bacon DR, Bylsma SS, Van Buskirk EM. Trabecular repopulation by anterior trabecular meshwork cells after laser trabeculoplasty. Am J Ophthalmol. 1989; 107(1):1-6.

28. Alexander RA, Grierson I. Morphological effects of argon laser trabeculoplasty upon the glaucomatous human meshwork. Eye (Lond). 1989; 3 (pt 6):719-726.

29. Alexander RA, Grierson I, Church WH. The effect of argon laser trabeculoplasty upon the normal human trabecular meshwork. Graefes Arch Clin Exp Ophthalmol. 1989;227(1):72-77.

30. Gonzalez P, Epstein DL, Luna C, Liton PB. Characterization of free-floating spheres from human trabecular meshwork (HTM) cell culture in vitro. Exp Eye Res. 2006;82(6):959-967.

31. Liton PB, Luna C, Challa P, Epstein DL, Gonzalez P. Genome-wide expression profile of human trabecular meshwork cultured cells, nonglaucomatous and primary open angle glaucoma tissue. $\mathrm{Mol}$ Vis. 2006;12:774-790.

32. Chen, M., et al. Generation of retinal ganglion-like cells from reprogrammed mouse fibroblasts [published online ahead of print May 19, 2010]. Invest Ophthalmol Vis Sci. doi:10.1167/iovs.09-4504.

33. Parameswaran $S$, et al. Induced pluripotent stem cells generate both retinal ganglion cells and photoreceptors: therapeutic implications in degenerative changes in glaucoma and agerelated macular degeneration. Stem Cells. 2010; 28(4):695-703

34. Jagatha $B$, et al. In vitro differentiation of reti- nal ganglion-like cells from embryonic stem cell derived neural progenitors. Biochem Biophys Res Commun. 2009;380(2):230-235.

35. Charwat $\mathrm{S}$, et al. Role of adult bone marrow stem cells in the repair of ischemic myocardium: current state of the art. Exp Hematol. 2008;36(6):672-680.

36. Petursson SR, Chervenick PA. Comparative effects of thrombopoietic-stimulatory factor and spleen cell-conditioned medium on megakaryocytopoiesis in a short-term bone marrow liquid culture system. Exp Hematol. 1988;16(8):660-666.

37. Sengupta N, et al. Regulation of adult hematopoietic stem cells fate for enhanced tissue-specific repair. Mol Ther. 2009;17(9):1594-1604.

38. Haspel RL, Miller KB. Hematopoietic stem cells: source matters. Curr Stem Cell Res Ther. 2008; 3(4):229-236

39. LeCouter J, et al. Angiogenesis-independent endothelial protection of liver: role of VEGFR-1. Science. 2003;299(5608):890-893.

40. Blazejewska EA, et al. Corneal limbal microenvironment can induce transdifferentiation of hair follicle stem cells into corneal epithelial-like cells. Stem Cells. 2009;27(3):642-652.

41. MacLaren RE, et al. Retinal repair by transplantation of photoreceptor precursors. Nature. 2006;444(7116):203-207.

42. Kustermann S, Hildebrandt H, Bolz S, Dengler K, Kohler K. Genesis of rods in the zebrafish retina occurs in a microenvironment provided by polysialic acid-expressing Muller glia. J Comp Neurol. 2010;518(5):636-646.

43. Banin E, et al. Retinal incorporation and differentiation of neural precursors derived from human embryonic stem cells. Stem Cells. 2006;24(2):246-257.

44. Li W, Ding S. Small molecules that modulate embryonic stem cell fate and somatic cell reprogramming. Trends Pharmacol Sci. 2010;31(1):36-45.

45. Asahara T, et al. Isolation of putative progenitor endothelial cells for angiogenesis. Science. 1997;275(5302):964-967.

46. Koike-Kiriyama N, et al. Human cord blood cells can differentiate into retinal nerve cells. Acta Neurobiol Exp (Wars). 2007;67(4):359-365.

47. Mezey E, Chandross KJ, Harta G, Maki RA, McKercher SR. Turning blood into brain: cells bearing neuronal antigens generated in vivo from bone marrow. Science. 2000;290(5497):1779-1782

48. Tropel $\mathrm{P}$, et al. Functional neuronal differentiation of bone marrow-derived mesenchymal stem cells. Stem Cells. 2006;24(12):2868-2876.

49. Roybon L, et al. Failure of transdifferentiation of adult hematopoietic stem cells into neurons. Stem Cells. 2006;24(6):1594-1604.

50. Snykers S, De Kock J, Rogiers V, Vanhaecke T. In vitro differentiation of embryonic and adult stem cells into hepatocytes: state of the art. Stem Cells. 2009;27(3):577-605.

51. Hill AJ, et al. Human umbilical cord blood-derived mesenchymal stem cells do not differentiate into neural cell types or integrate into the retina after intravitreal grafting in neonatal rats. Stem Cells Dev. 2009;18(3):399-409.

52. Chiou SH, et al. A novel in vitro retinal differ- 
entiation model by co-culturing adult human bone marrow stem cells with retinal pigmented epithelium cells. Biochem Biophys Res Commun. 2005;326(3):578-585

53. Gill $\mathrm{M}$, et al. Vascular trauma induces rapid but transient mobilization of VEGFR2(+)AC133(+) endothelial precursor cells. Circ Res. 2001; 88(2):167-174

54. Kalka C, et al. VEGF gene transfer mobilizes endothelial progenitor cells in patients with inoperable coronary disease. Ann Thorac Surg. 2000; $70(3): 829-834$

55. Kocher AA, et al. Neovascularization of ischemic myocardium by human bone-marrow-derived angioblasts prevents cardiomyocyte apoptosis, reduces remodeling and improves cardiac function. Nat Med. 2001;7(4):430-436.

56. Grant MB, et al. Adult hematopoietic stem cells provide functional hemangioblast activity during retinal neovascularization. Nat Med. 2002;8(6):607-612.

57. Blau H, Brazelton T, Keshet G, Rossi F. Something in the eye of the beholder. Science. 2002; 298(5592):361-362.

58 . Krause DS. Plasticity of marrow-derived stem cells. Gene Ther. 2002;9(11):754-758.

59. Barber CL, Iruela-Arispe ML. The ever-elusive endothelial progenitor cell: identities, functions and clinical implications. Pediatr Res. 2006; 59(4 pt 2):26R-32R.

60. Espinosa-Heidmann DG, Caicedo A, Hernandez EP, Csaky KG, Cousins SW. Bone marrow-derived progenitor cells contribute to experimental choroidal neovascularization. Invest Ophthalmol Vis Sci. 2003;44(11):4914-4919.

61. Sengupta N, Caballero S, Mames RN, Butler JM, Scott EW, Grant MB. The role of adult bone marrowderived stem cells in choroidal neovascularization. Invest Ophthalmol Vis Sci. 2003;44(11):4908-4913.

62. Elsheikh E, Uzunel M, He Z, Holgersson J, Nowak G, Sumitran-Holgersson S. Only a specific subset of human peripheral-blood monocytes has endothelial-like functional capacity. Blood. 2005; 106(7):2347-2355

63. Fujiyama $\mathrm{S}$, et al. Bone marrow monocyte lineage cells adhere on injured endothelium in a monocyte chemoattractant protein-1-dependent manner and accelerate reendothelialization as endothelial progenitor cells. Circ Res. 2003 ; 93(10):980-989.

64. Otani A, Kinder K, Ewalt K, Otero FJ, Schimmel $\mathrm{P}$, Friedlander M. Bone marrow-derived stem cells target retinal astrocytes and can promote or inhibit retinal angiogenesis. Nat Med. 2002; 8(9):1004-1010.

65. Dorrell MI, Otani A, Aguilar E, Moreno SK, Friedlander M. Adult bone marrow-derived stem cells use R-cadherin to target sites of neovascularization in the developing retina. Blood. 2004; 103(9):3420-3427.

66. Otani A, et al. Rescue of retinal degeneration by intravitreally injected adult bone marrow-derived lineage-negative hematopoietic stem cells. J Clin Invest. 2004;114(6):765-774.

67. Otani A, et al. A fragment of human TrpRS as a potent antagonist of ocular angiogenesis. Proc Natl Acad Sci U S A. 2002;99(1):178-183.

68. Ritter MR, Banin E, Moreno SK, Aguilar E, Dorrell MI, Friedlander M. Myeloid progenitors differentiate into microglia and promote vascular repair in a model of ischemic retinopathy. J Clin Invest. 2006;116(12):3266-3276

69. Higashi Y, et al. Autologous bone-marrow mononuclear cell implantation improves endotheliumdependent vasodilation in patients with limb ischemia. Circulation. 2004;109(10):1215-1218

70. Checchin D, Sennlaub F, Levavasseur E, Leduc M, Chemtob $\mathrm{S}$. Potential role of microglia in retinal blood vessel formation. Invest Ophthalmol Vis Sci.
2006;47(8):3595-3602.

71. Galimi F, Summers RG, van Praag H, Verma IM, Gage FH. A role for bone marrow-derived cells in the vasculature of noninjured CNS. Blood. 2005;105(6):2400-2402.

72. Zeng HY, Green WR, Tso MO. Microglial activation in human diabetic retinopathy. Arch Ophthalmol. 2008;126(2):227-232.

73. Urashima M, et al. Umbilical cord blood as a rich source of immature hematopoietic stem cells. Acta PaediatrJpn. 1994;36(6):649-655.

74. Harris DT, Rogers I. Umbilical cord blood: a unique source of pluripotent stem cells for regenerative medicine. Curr Stem Cell Res Ther. 2007; 2(4):301-309.

75. Konuma T, et al. Myeloablative unrelated cord blood transplantation for acute leukemia patients between 50 and 55 years of age: single institutional retrospective comparison with patients younger than 50 years of age. Ann Hematol. 2009; 88(6):581-588.

76. Hildbrand $\mathrm{P}$, et al. The role of angiopoietins in the development of endothelial cells from cord blood CD34+ progenitors. Blood. 2004; 104(7):2010-2019.

77. Crisa L, Cirulli V, Smith KA, Ellisman MH Torbett BE, Salomon DR. Human cord blood progenitors sustain thymic T-cell development and a novel form of angiogenesis. Blood. 1999; 94(11):3928-3940.

78. Chen J, Smith LE. Retinopathy of prematurity. Angiogenesis. 2007;10(2):133-140.

79. Hoefer IE, et al. Leukocyte subpopulations and arteriogenesis: specific role of monocytes, lymphocytes and granulocytes. Atherosclerosis. 2005;181(2):285-293

80. Schruefer R, Lutze N, Schymeinsky J, Walzog B. Human neutrophils promote angiogenesis by a paracrine feedforward mechanism involving endothelial interleukin-8. Am J Pbysiol Heart Circ Physiol. 2005;288(3):H1186-1192.

81. Kusumanto YH, Dam WA, Hospers GA, Meijer C, Mulder NH. Platelets and granulocytes, in particular the neutrophils, form important compartments for circulating vascular endothelial growth factor. Angiogenesis. 2003;6(4):283-287.

82. Mantovani A, Sozzani S, Locati M, Allavena P, Sica A. Macrophage polarization: tumor-associated macrophages as a paradigm for polarized M2 mononuclear phagocytes. Trends Immunol. 2002;23(11):549-555

83. Mantovani A, Sica A, Sozzani S, Allavena P, Vecchi A, Locati M. The chemokine system in diverse forms of macrophage activation and polarization. Trends Immunol. 2004;25(12):677-686.

84. Brichard B, et al. Intracellular cytokine profile of cord and adult blood monocytes. Bone Marrow Transplant. 2001;27(10):1081-1086.

85. Gustafsson C, et al. Gene expression profiling of human decidual macrophages: evidence for immunosuppressive phenotype. PLoS One. 2008;3(4):e2078.

86. Congdon N, et al. Causes and prevalence of visual impairment among adults in the United States. Arch Ophthalmol. 2004;122(4):477-485.

87. Resnikoff S, et al. Global data on visual impairment in the year 2002. Bull World Health Organ. 2004;82(11):844-851.

88. Friedman DS, et al. Prevalence of age-related macular degeneration in the United States. Arch Ophthalmol. 2004;122(4):564-572

89. Zarbin MA. Current concepts in the pathogenesis of age-related macular degeneration. Arch Ophthalmol. 2004;122(4):598-614.

90. de Jong PT. Age-related macular degeneration. N Engl J Med. 2006;355(14):1474-1485.

91 . Strauss O. The retinal pigment epithelium in visual function. Physiol Rev. 2005;85(3):845-881.

92. Rosenfeld PJ, et al. Ranibizumab for neovascular age-related macular degeneration. $N$ Engl J Med. 2006;355(14):1419-1431.

93. Brown DM, et al. Ranibizumab versus verteporfin for neovascular age-related macular degeneration. N Engl J Med. 2006;355(14):1432-1444.

94. Algvere PV, Gouras P, Dafgard Kopp E. Long-term outcome of RPE allografts in non-immunosuppressed patients with AMD. Eur J Ophthalmol. 1999;9(3):217-230.

95. Zhang X, Bok D. Transplantation of retinal pigment epithelial cells and immune response in the subretinal space. Invest Ophthalmol Vis Sci. 1998;39(6):1021-1027.

96. Malone PE, Herndon LW, Muir KW, Jaffe GJ. Combined fluocinolone acetonide intravitreal insertion and glaucoma drainage device placement for chronic uveitis and glaucoma. Am J Ophthalmol. 2010;149(5):800-806.

97. Cahill MT, Freedman SF, Toth CA. Macular translocation with 360 degrees peripheral retinectomy for geographic atrophy. Arch Ophthalmol. 2003;121(1):132-133.

98. Joussen AM, et al. Autologous translocation of the choroid and retinal pigment epithelium in patients with geographic atrophy. Ophthalmology. 2007;114(3):551-560.

99. Klimanskaya I, Hipp J, Rezai KA, West M, Atala A, Lanza R. Derivation and comparative assessment of retinal pigment epithelium from human embryonic stem cells using transcriptomics. Cloning Stem Cells. 2004;6(3):217-245.

100.Lund RD, et al. Human embryonic stem cellderived cells rescue visual function in dystrophic RCS rats. Cloning Stem Cells. 2006;8(3):189-199.

101.Vugler A, et al. Elucidating the phenomenon of HESC-derived RPE: anatomy of cell genesis, expansion and retinal transplantation. Exp Neurol. 2008;214(2):347-361.

102. Meyer JS, et al. Modeling early retinal development with human embryonic and induced pluripotent stem cells. Proc Natl Acad Sci U S A. 2009;106(39):16698-16703.

103. Carr AJ, et al. Molecular characterization and functional analysis of phagocytosis by human embryonic stem cell-derived RPE cells using a novel human retinal assay. Mol Vis. 2009; 15:283-295.

104.Gong J, Sagiv O, Cai H, Tsang SH, Del Priore LV. Effects of extracellular matrix and neighboring cells on induction of human embryonic stem cells into retinal or retinal pigment epithelial progenitors. Exp Eye Res. 2008;86(6):957-965

105.Idelson $\mathrm{M}$, et al. Directed differentiation of human embryonic stem cells into functional retinal pigment epithelium cells. Cell Stem Cell. 2009;5(4):396-408.

106. Osakada F, Ikeda H, Sasai Y, Takahashi M. Stepwise differentiation of pluripotent stem cells into retinal cells. Nat Protoc. 2009;4(6):811-824.

107. Osakada F, et al. In vitro differentiation of retinal cells from human pluripotent stem cells by small-molecule induction. J Cell Sci. 2009;122(Pt 17):3169-3179.

108. Takahashi K, et al. Induction of pluripotent stem cells from adult human fibroblasts by defined factors. Cell. 2007;131(5):861-872.

109.Yu J, et al. Induced pluripotent stem cell lines derived from human somatic cells. Science. 2007;318(5858):1917-1920.

110. Buchholz DE, et al. Derivation of functional retinal pigmented epithelium from induced pluripotent stem cells. Stem Cells. 2009;27(10):2427-2434.

111. Carr AJ, et al. Protective effects of human iPSderived retinal pigment epithelium cell transplantation in the retinal dystrophic rat. PLoS One. 2009;4(12):e8152.

112.Hirami Y, et al. Generation of retinal cells from mouse and human induced pluripotent stem cells. Neurosci Lett. 2009;458(3):126-131. 
113. Yu J, et al. Human induced pluripotent stem cells free of vector and transgene sequences. Science. 2009;324(5928):797-801.

114.Kim D, et al. Generation of human induced pluripotent stem cells by direct delivery of reprogramming proteins. Cell Stem Cell. 2009;4(6):472-476.

115.Li W, et al. Generation of human-induced pluripotent stem cells in the absence of exogenous Sox2. Stem Cells. 2009;27(12):2992-3000.

116.Nakagawa M, et al. Generation of induced pluripotent stem cells without Myc from mouse and human fibroblasts. Nat Biotechnol. 2008; 26(1):101-106.

117. Inoue T, et al. Maximizing functional photoreceptor differentiation from adult human retinal stem cells. Stem Cells. 2010;28(3):489-500.

118. Tropepe V, et al. Retinal stem cells in the adult mammalian eye. Science. 2000;287(5460):2032-2036.

119. Cicero SA, et al. Cells previously identified as retinal stem cells are pigmented ciliary epithelial cells. Proc Natl Acad Sci U S A. 2009;106(16):6685-6690.

120.Gamm DM, et al. Protection of visual functions by human neural progenitors in a rat model of retinal disease. PLoS One. 2007;2(3):e338.

121. Dorrell MI, et al. Antioxidant or neurotrophic factor treatment preserves function in a mouse model of neovascularization-associated oxidative stress. J Clin Invest. 2009;119(3):611-623.

122.Davison AC. Brave pioneers or clinical cowboys? Cell Stem Cell. 2010;6(6):504-505.

123. Marc RE, Jones BW, Watt CB, Strettoi E. Neural remodeling in retinal degeneration. Prog Retin Eye Res. 2003;22(5):607-655. 